\title{
Высокотемпературная диффузия магния в бездислокационном кремнии
}

\author{
(С В.Б. Шуман, Ю.А. Астров, А.Н. Лодыгин, Л.М. Порцель
}

Физико-технический институт им. А.Ф. Иоффре Российской академии наук, 194021 Санкт-Петербург, Россия

E-mail: Shuman@mail.ioffe.ru

(Получена 31 января 2017 г. Принята к печати 15 февраля 2017 г.)

С использованием сандвич-метода легирования монокристаллического бездислокационного кремния магнием исследована диффузия примеси в диапазоне температур $1000-1200^{\circ} \mathrm{C}$. Найденная зависимость коэффициента диффузии межузельного магния от температуры универсальным образом описывает данные настоящей работы и ранее исследованную температурную область $600-800^{\circ} \mathrm{C}$. Малая энергия активации диффузии (1.83 эВ) свидетельствует о межузельном транспорте примеси.

DOI: $10.21883 /$ FTP.2017.08.44792.8532

\section{1. Введение}

Кремний, легированный глубокими двойными донорами, такими как $\mathrm{S}, \mathrm{Se}, \mathrm{Mg}$, представляет собой перспективный материал для исследований в области квантовых технологий [1-3]. Электрически активный магний занимает особое место среди указанных примесей. В отличие от других элементов II группы (Be, Zn), он находится в межузельных положениях в решетке кремния. Энергии уровней магния составляют $E_{C}-0.107$ эВ для нейтрального состояния $\mathrm{Mg}^{0}$ и $E_{C}-0.256$ эВ для $\mathrm{Mg}^{+}[4,5]$.

Для ряда задач (таких, например, как исследование динамики внутрицентровых переходов $\mathrm{Mg}^{0}$ [3]) требуются однородно легированные образцы с достаточными объемом и концентрацией примесных центров. Как правило, $\mathrm{Mg}$ вводится в кремний с помощью „сандвичметода“ диффузии (sandwich diffusion technique) в диапазоне температур $T=1000-1200^{\circ} \mathrm{C}[4,5]$, такой метод позволяет получать образцы с концентрацией примеси до $N_{\mathrm{Mg}} \approx 3 \cdot 10^{15} \mathrm{~cm}^{-3}$. Хотя ряд свойств $\mathrm{Si}: \mathrm{Mg}$ к настоящему времени хорошо изучен, ощущается недостаток данных о таких важных для материаловедения параметpax, как растворимость электрически активного магния и температурная зависимость коэффициента диффузии.

Количественные данные о коэффициенте диффузии $D_{\mathrm{Mg}}$ электрически активного $\mathrm{Mg}$ в $\mathrm{Si}$ были впервые получены в работе [6], где была найдена температурная зависимость $D_{\mathrm{Mg}}(T)$ в диапазоне $T=600-800^{\circ} \mathrm{C}$. Коэффициент диффузии примеси был изучен в работе [6] с применением метода ионного легирования поверхности пластин $p$-Si магнием и последующей термообработки образцов при различных температурах и длительностях. Значения $D_{M g}$ находили из измерений глубины залегания $p-n$-перехода, образующегося в такой структуре.

Настоящее исследование посвящено определению коэффициента диффузии магния в кремнии в температурном диапазоне $T=1000-1200^{\circ} \mathrm{C}$. В экспериментах использовали традиционный сандвич-метод легирования материала. Коэффициент диффузии определяли путем анализа пространственных распределений электрически активной примеси $\mathrm{Mg}$ по глубине образцов, полученных методом измерения дифференциальной проводимости. Результаты работы сопоставляются с ранее полученной в работе [6] зависимостью $D_{\mathrm{Mg}}(T)$ в низкотемпературной области.

\section{2. Методика эксперимента}

Диффузию $\mathrm{Mg}$ в $\mathrm{Si}$ проводили сандвич-методом при различных температурах и временах. В качестве исходного материала использовали пластины бестигельного $p$-Si c удельным сопротивлением $2.0 \cdot 10^{3} \mathrm{OM} \cdot \mathrm{cm}$ (разностная концентрация акцепторов и доноров $\left.6.3 \cdot 10^{12} \mathrm{~cm}^{-3}\right)$, ориентацией $(111)$, толщиной $d=1.5-2$ мм и диаметром 30 мм. На одну сторону образца распылением в вакууме наносили слой магния толщиной $1.2-1.5$ мкм. Использовали $\mathrm{Mg}$ чистотой 99.999\% от различных производителей (Goodfellow и Mateck). Затем со стороны слоя $\mathrm{Mg}$ к образцу прижимали дополнительную пластинку кремния толщиной $d=0.5$ мм. Такую сандвич-конструкцию устанавливали в кварцевую кассету. Кассету помещали в кварцевую ампулу, которую заполняли аргоном и заваривали. При высокой температуре диффузионного процесса вспомогательная пластинка спаивалась с образцом, что препятствовало испарению магния.

Диффузию проводили при $T=1000-1200^{\circ} \mathrm{C}$ в течение $t=0.5-2.5$ ч. После окончания процесса ампулу принудительно охлаждали в потоке сжатого воздуха. Материал дополнительной пластинки сандвича удаляли шлифовкой. Распределение концентрации примеси по глубине образца определяли методом дифференциальной проводимости. Для получения данных с образца последовательно удаляли слои толщиной $\sim 20$ мкм. При этом с помощью четырехзондового метода измеряли сопротивление образца до и после удаления слоя. Полученное из этих измерений распределение удельной проводимости образца по глубине аппроксимировали аналитической функцией, которую затем использовали 
для расчета профилей концентрации электронов $n$ и примесных центров $N_{\mathrm{Mg}}$.

Как отмечено выше, магний в кремнии является двухзарядным донором. При комнатной температуре почти все центры находятся в состоянии $\mathrm{Mg}^{+}$и частично в состоянии $\mathrm{Mg}^{++}$. Для вычисления $N_{\mathrm{Mg}}$ из величины $n$ мы использовали подход, который применили в работе [7] при обработке результатов измерений эффекта Холла: в каждой точке профиля концентрации электронов, найденного для комнатной температуры, была определена величина $N_{\mathrm{Mg}}$. Это достигалось путем численного решения уравнения электронейтральности полупроводника, легированного глубокой донорной примесью.

Полученную зависимость $N_{\mathrm{Mg}}(x)$ далее использовали для нахождения коэффициента диффузии магния путем подгонки теоретической зависимости, которая описывает форму профиля примеси в случае диффузии из бесконечного источника [8],

$$
N_{\mathrm{Mg}}(x, t)=N_{S} \operatorname{erfc}\left(\frac{x}{2 \sqrt{D_{\mathrm{Mg}} t}}\right),
$$

к экспериментальной кривой. В (1) $N_{S}$ - концентрация на поверхности, которая полагается фиксированной в течение диффузионного процесса, $x$ - расстояние от поверхности образца, $t$ - время диффузии, erfc - дополнительная функция ошибок Гаусса (Gauss complementary error function). Коэффициент диффузии в выражении (1) служил подгоночным параметром к данным экспериментов.

\section{3. Экспериментальные результаты и обсуждение}

На рис. 1 представлены профили распределения концентрации $N_{\mathrm{Mg}}$, полученные для диффузионного процес-

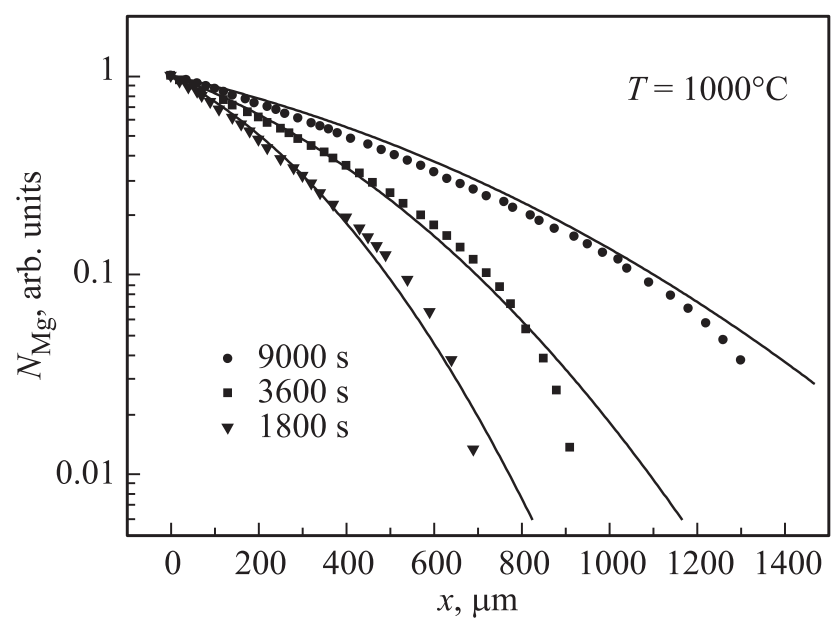

Рис. 1. Профили концентрации межузельного магния для диффузионного процесса в течение 1800,3600 и 9000 с при $T=1000^{\circ} \mathrm{C}$. Сплошная кривая - расчет согласно $(1)$ при $D_{\mathrm{Mg}}=2.5 \cdot 10^{-7} \mathrm{~cm}^{2} \cdot \mathrm{c}^{-1}$ и соответствующих временах.

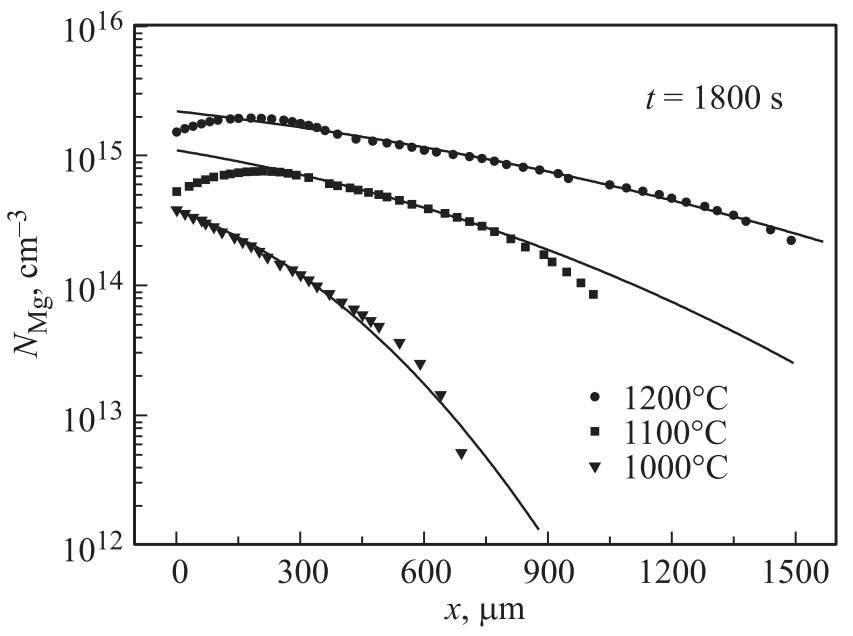

Рис. 2. Пространственные распределения межузельного магния при диффузии в течение 1800 с и различных температурах. Сплошные кривые - расчет согласно уравнению (1) с коэффициентом диффузии $D_{\mathrm{Mg}}=2.5 \cdot 10^{-7}$, $1.2 \cdot 10^{-6}, 2.5 \cdot 10^{-6} \mathrm{~cm}^{2} \cdot \mathrm{c}^{-1}$ для $T=1000,1100,1200^{\circ} \mathrm{C}$ соответственно.

са при $T=1000^{\circ} \mathrm{C}$ и различных временах диффузии $t=1800,3600$ и 9000 с. Экспериментальные профили представлены в нормированном виде. Сплошными линиями показаны построенные с использованием уравнения (1) распределения, наилучшим образом описывающие данные экспериментов. Как видно из этих данных, используемая функция erfc достаточно хорошо описывает экспериментальные профили введенной примеси для всех значений времени диффузии, причем совокупность данных удовлетворительно описывается при использовании единого значения $D_{\mathrm{Mg}}=2.5 \cdot 10^{-7} \mathrm{~cm}^{2} \cdot \mathrm{c}^{-1}$.

На рис. 2 показаны данные для условий изохронного процесса диффузии: профили распределения примеси относятся к различным температурам $(T=1000,1100$ и $1200^{\circ} \mathrm{C}$ ) при времени диффузии $t=30$ мин. Как и на рис. 1, сплошными линиями показаны расчетные профили. Коэффициенты диффузии, определенные для этих температур, составили $D_{\mathrm{Mg}}=2.5 \cdot 10^{-7}, 1.2 \cdot 10^{-6}$ и $2.5 \cdot 10^{-6} \mathrm{~cm}^{2} \cdot \mathrm{c}^{-1}$ соответственно.

Представленные на рис. 1,2 данные свидетельствуют, что общий ход спада концентрации примеси вглубь образцов достаточно хорошо описывается зависимостью (1). Вместе с тем существует закономерность в пространственных распределениях продиффундировавшей примеси: в глубине образцов наблюдается более быстрый спад концентрации, чем это описывается выражением (1). Кроме того, полученные при высоких температурах $\left(T=1100\right.$ и $\left.1200^{\circ} \mathrm{C}\right)$ профили обнаруживают выраженные особенности в приповерхностной области образца глубиной $~ 300$ мкм. Обе указанные особенности, наблюдающиеся в процессе диффузии, требуют дальнейшего изучения.

На рис. 3 представлены значения коэффициента диффузии, определенные в настоящей работе, а также 


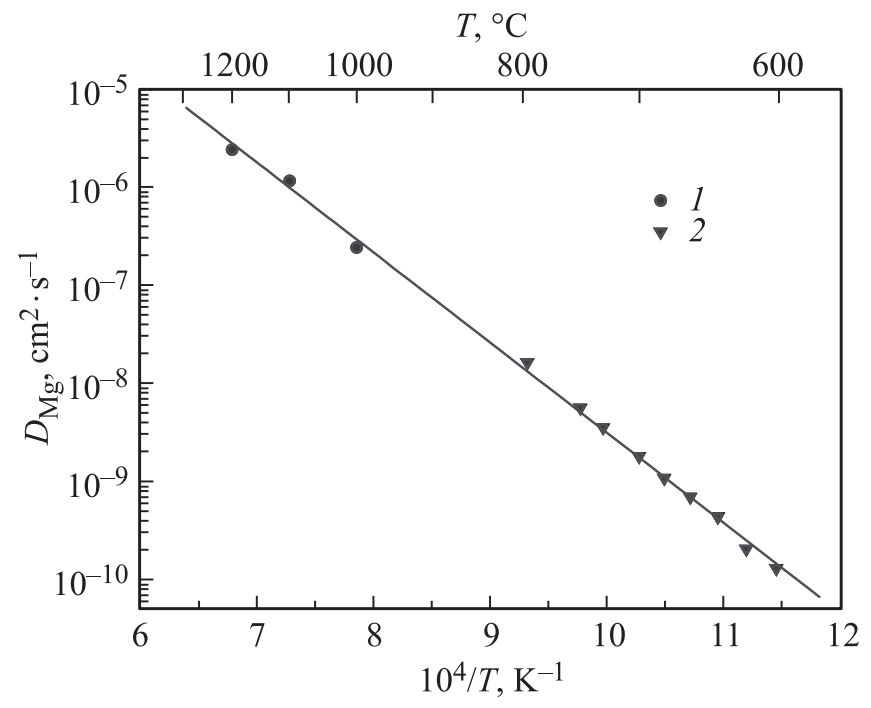

Рис. 3. Температурная зависимость $D_{\mathrm{Mg}}$, полученная при легировании сандвич-методом в настоящей работе (1) и в экспериментах [6] на ионно-имплантированных слоях (2). Сплошная линия - зависимость Аррениуса (2).

данные для $D_{\mathrm{Mg}}$, полученные в работе [6] в диапазоне температур $T=600-800^{\circ} \mathrm{C}$ с использованием ионноимплантированных образцов. Как видно, экспериментальные значения $D_{\mathrm{Mg}}$, измеренные разными методами в температурном диапазоне $600-1200^{\circ} \mathrm{C}$, хорошо укладываются на зависимость Аррениуса:

$$
D_{\mathrm{Mg}}=5.3 \exp \left(-E_{a} / k_{\mathrm{B}} T\right)
$$

где энергия активации $E_{a}=1.83$ эВ, $k_{\mathrm{B}}-$ постоянная Больцмана, $T-$ температура, $D_{M g}$ измеряется в $\mathrm{cm}^{2} \cdot \mathrm{c}^{-1}$. Малая энергия активации характерна для диффузии по междоузлиям.

Низкотемпературная абсорбционная фурье-спектроскопия образцов, легированных сандвич-методом, обнаруживает в них следы лития. Использование известных данных о сечении поглощения этой примеси в кремнии позволяет оценить ее концентрацию, которая обычно составляет $<10^{13} \mathrm{~cm}^{-3}$. Использование магния различных производителей не позволило избавиться от примеси лития в образцах. По-видимому, некоторое количество лития содержится в магнии высокой чистоты, который использовали в опытах. Мы полагаем, что, являясь быстродиффундирующей примесью в кремнии, литий практически однородно заполняет объем образца для исследованных в работе режимов диффузии и не оказывает существенного влияния на форму измеряемых профилей магния. При ионном легировании образцов, использованном в работе [6], введение лития в образцы исключается. Таким образом, косвенным свидетельством малой роли лития в получении данных настоящей работы является то, что кривая Аррениуса (рис. 3) универсальным образом описывает низко- и высокотемпературную области.
Авторы выражают благодарность С.Г. Павлову за измерение спектров поглощения образцов $\mathrm{Si}: \mathrm{Mg}$ при низких температурах.

\section{Список литературы}

[1] K.J. Morse, R.J.S. Abraham, H. Riemann, N.V. Abrosimov, P. Becker, H.-J. Pohl, M.L.W. Thewalt, S. Simmons. arXiv:1606.03488v1 [quant-ph] 10 June 2016.

[2] N.V. Abrosimov, N. Nötzel, H. Riemann, K. Irmscher, S.G. Pavlov, H.-W. Hübers, U. Böttger, P.M. Haas, N. Drichko, M. Dressel. Sol. St. Phenomena, 131-133, 589 (2008).

[3] S.G. Pavlov, N. Deßmann, A. Pohl, V.B. Shuman, L.M. Portsel, A.N. Lodygin, Yu.A. Astrov, S. Winnerl, H. Schneider, N. Stavrias, A.F.G. van der Meer, V.V. Tsyplenkov, K.A. Kovalevsky, R.Kh. Zhukavin, V.N. Shastin, N.V. Abrosimov, H.-W. Hübers. Phys. Rev. B, 94, 075208 (2016).

[4] R.F. Franks, J.B. Robertson. Solid State Commun., 5, 479 (1967).

[5] L.T. Ho, A.K. Ramdas. Phys. Rev. B, 5, 462 (1972).

[6] В.Б. Шуман, А.А. Лаврентьев, Ю.А. Астров, А.Н. Лодыгин, Л.М. Порцель. ФТП, 51 (1), 6 (2017).

[7] Yu.A. Astrov, L.M. Portsel, A.N. Lodygin, V.B. Shuman. Semicond. Sci. Technol., 26, 055021 (2011).

[8] Технология СБИС, под ред. С. Зи (М., Мир, 1986) т. 1.

Редактор Л.В. Шаронова

\section{High-temperature magnesium diffusion in dislocation-free silicon}

\section{V.B. Shuman, Yu.A. Astrov, A.N. Lodygin, L.M. Portsel}

loffe Institute, 194021 St. Petersburg, Russia

Abstract Diffusion doping of dislocation-free single-crystal silicon with magnesium is investigated in the temperature range $1000-1200^{\circ} \mathrm{C}$. The traditional sandwich method is applied as a method of doping. It is found that the diffusion coefficient of magnesium is described by a universal Arrhenius curve in the extended temperature range $600-1200^{\circ} \mathrm{C}$, when data obtained earlier for the interval $600-800^{\circ} \mathrm{C}$ are taken into consideration. A relatively low activation energy of diffusion transport $(1.83 \mathrm{eV})$ gives an evidence of interstitial mechanism of the impurity diffusion. 